\title{
Preparation, characterization and investigation of the anti-human ovarian cancer activity of silver nanoparticles green-formulated by Salvia officinalis leaf aqueous extract
}

\author{
Danxia Luo ${ }^{1}$, Arunachalam Chinnathambi², Tahani Awad Alahmadi ${ }^{3}$ D.S. Prabakaran ${ }^{4}$, \\ Gaofeng Zhang 5
}

\author{
${ }^{1}$ Department of Gynecology, Shaanxi Provincial People's Hospital, Xi'an, China \\ ${ }^{2}$ Department of Botany and Microbiology, College of Science, King Saud University, \\ Riyadh, Saudi Arabia \\ ${ }^{3}$ Department of Pediatrics, College of Medicine and King Khalid University Hospital, \\ King Saud University, Riyadh, Saudi Arabia \\ ${ }^{4}$ Department of Radiation Oncology, Chungbuk National University, \\ College of Medicine, Cheongju, Korea \\ ${ }^{5}$ Project Department, Zhongke Inno International Medical Research Institute, Beijing, \\ China
}

Submitted: 9 July 2021; Accepted: 18 December 2021

Online publication: 6 January 2022

Arch Med Sci

DOI: https://doi.org/10.5114/aoms/145111

Copyright (c) 2022 Termedia \& Banach

\begin{abstract}
Introduction: In the present study, we decided to prepare and formulate a new chemotherapeutic drug (silver nanoparticles in aqueous medium using Salvia officinalis leaf aqueous extract) for the treatment of human ovarian cancer in in vitro conditions.

Material and methods: Organometallic chemistry methods such as scanning electron microscopy (SEM), UV-visible spectroscopy (UV-Vis), and Fourier transformed infrared spectroscopy (FT-IR) were used to characterize silver nanoparticles. To investigate the antioxidant potential of $\mathrm{AgNO}_{3}$, Salvia officinalis aqueous extract, and silver nanoparticles, the DPPH test was used in the presence of butylated hydroxytoluene as the positive control. To survey the cytotoxicity and anti-human ovarian cancer activities of $\mathrm{AgNO}_{3}$, Salvia officinalis aqueous extract, and silver nanoparticles, MTT assay was used on human ovarian cancer cell lines i.e., Caov-3, SK-OV-3, and PA-1.

Results: In UV-Vis, the clear peak at the wavelength of $421 \mathrm{~nm}$ indicated the formation of silver nanoparticles. In the FT-IR test, the presence of many antioxidant compounds with related bonds created excellent conditions for reducing silver in the silver nanoparticles. The silver nanoparticles inhibited half of the DPPH molecules at the concentration of $251 \mu \mathrm{g} / \mathrm{ml}$.

Conclusions: The best result of anti-human ovarian cancer effects of silver nanoparticles against the above cell lines was observed in the case of the SK-OV-3 cell line. Silver nanoparticles had very low cell viability and anti-human ovarian cancer properties dose-dependently against Caov-3, SK-OV-3, and PA-1 cell lines without any cytotoxicity towards the normal cell line (HUVECS).
\end{abstract}

Key words: Salvia officinalis leaf, silver nanoparticles, human ovarian cancer effects.

\author{
Corresponding author: \\ Gaofeng Zhang \\ Project Department \\ Zhongke Inno \\ International Medical \\ Research Institute \\ Beijing, China \\ E: mail: zgf23681@163.com
}




\section{Introduction}

The ovary is an organ found in the female reproductive system that produces an ovum. When released, this travels down the fallopian tube into the uterus, where it may become fertilized by a sperm. There is an ovary found on each side of the body. The ovaries also secrete hormones that play a role in the menstrual cycle and fertility. The ovary progresses through many stages from the prenatal period to menopause. It is also an endocrine gland because of the various hormones that it secretes [1]. The main diseases that affect the normal function of the ovary include endometriosis, ovarian cysts, ovarian germ cell tumors, ovarian low malignant potential tumors, polycystic ovary syndrome, and ovarian epithelial cancer (ovarian cancer) [1, 2]. Ovarian cancer is one of the most common cancers in women in both developing and developed countries. At first, ovarian cancer arises with growth of an abnormal cell and after a while it spreads to all parts of the uterine and also all parts of the body [2,3]. Ovarian cancer is a cancer that forms in or on an ovary. It results in abnormal cells that have the ability to invade or spread to other parts of the body. When this process begins, there may be no or only vague symptoms. Symptoms become more noticeable as the cancer progresses. The symptoms of ovarian cancer are pain or pressure in the pelvis, pain in the back or abdomen, feeling full rapidly when eating, changes in bowel habits such as constipation, unexpected vaginal bleeding, bloating, changes in urination patterns such as more frequent urination, and maybe nausea and indigestion, appetite loss, weight loss, breathlessness, and fatigue [3, 4]. The main risk factors of ovarian cancer are family history, age, reproductive history, breast cancer, hormone therapy, obesity and overweight, gynecologic surgery, human papillomavirus, and the use of talcum powder [1, 2]. Suppression of ovulation, which would otherwise cause damage to the ovarian epithelium and, consequently, inflammation, is generally protective. This effect can be achieved by having children, taking combined oral contraceptives, and breast feeding, all of which are protective factors. A longer period of breastfeeding correlates with a larger decrease in the risk of ovarian cancer. Each birth decreases risk of ovarian cancer more, and this effect is seen with up to five births [1-4]. Blood tests, imaging examination, laparoscopy, and biopsy are used for the diagnosis of ovarian cancers in the several stages of cancer including localized, regional, and distant $[4,5]$. For the treatment of ovarian cancer, chemotherapy, radiation therapy, and immunotherapy are used $[5,6]$. Due to the considerable side effects of the above therapeutic methods, the formulation of modern chemotherapeutic drugs is necessary [4, 5]. Recently, scientists have discovered that metallic nanoparticles have excellent anticancer properties. Metallic nanoparticles have received considerable attention in the field of medicine. Recently, the metal-based nanoparticles with distinctive physicochemical properties have been considered as a promising alternative medicine for bacterial disease and cancer treatment [4-6].

Due to the recent advances in research on metallic nanoparticles, silver nanoparticles are effective antifungal agents due to their powerful cytotoxic activity toward a broad range of microorganisms compared to other metals [7, 8]. Silver nanoparticles have drawn significant attention because of their wide applications in chemical, electrical, optical, bioremediation, sensor, and biological fields [6, 7]. Silver nanoparticles green-synthesized using medicinal plants have been abundantly used in the biomedical sciences for the treatment of many diseases. Every year, notable applications of green-synthesized silver nanoparticles are being achieved, and this trend is continuing [8-10]. The results of many studies have indicated the significant antifungal effects of silver nanoparticles green-synthesized by plants in the cure of candida diseases and their antibacterial properties in the treatment of Streptococcus, Staphylococcus, Pseudomonas, Salmonella, and Bacillus infectious. Also, silver nanoparticles synthesized by plants have been formulated due to the antiviral, antibacterial, antioxidant, anti-parasitic, anti-inflammatory, antifungal, wound healing, and anti-cancer properties [8].

The main therapeutic properties of metallic nanoparticles are anticancer effects. A study reported the anti-human breast cancer properties of metallic nanoparticles in the cellular and molecular conditions. In the previous study, metallic nanoparticles significantly killed all malignant breast cells (MCF-7 cells) in nano concentrations. In another study, metallic nanoparticles synthesized using benzimidazole indicated the remarkable anticancer effects against malignant cancer cells, i.e. A549 and NIH/3T3 cell lines [11]. Chatterjee et al. reported that metallic nanoparticles in nano concentrations had excellent anticancer potentials against rat glioblastoma C6-G, human lung cancer A-549, and human skin melanoma A-375 [12] Azizi et al. recorded excellent anticancer activities of metallic nanoparticles against normal MCF-10A counterparts and invasive MDA-MB 231 cancer cells [13]. Also, regarding the anticancer properties of metallic nanoparticles green-synthesized by plants, Ramaswamy et al. reported that when metallic salt was combined with medicinal plants, many anticancer supplements with significant effects against MCF-7 breast cancer cells were made [14]. Also, Sumathi et al. [15] and Roberson et al. 
[16] mentioned notable anticancer properties of metallic nanoparticles containing Prosopis cineraria against human MCF-7 and HeLa cancer cells. In other studies, the anticancer properties of metallic nanoparticles green-synthesized by Nerium oleander [17], Magnolia kobus [18], and Eclipta prostrata [19] were confirmed against the human HepG2 cancer cell line.

In the present study, we decided to investigate the anti-human ovarian cancer potential of silver nanoparticles formulated by Salvia officinalis leaf aqueous extract against the common human ovarian cancer cell lines Caov-3, SK-OV-3, and PA-1.

\section{Material and methods}

\section{Material}

Dimethyl sulfoxide (DMSO), antimycotic antibiotic solution, hydrolysate, fetal bovine serum, Ehrlich solution, 4-(dimethylamino)benzaldehyde, 2,2-diphenyl-1-picrylhydrazyl (DPPH), carbazole reagent, borax-sulfuric acid mixture, Dulbecco's Modified Eagle Medium (DMED), and phosphate buffer solution (PBS) were all obtained from Sigma-Aldrich (USA).

\section{Preparation of aqueous extract of Salvia officinalis leaf}

Salvia officinalis was identified by verifying the color pictures followed by description and identification of characters. The plant peels were thoroughly washed with tap water to remove dust and other unwanted materials accumulated on the peels from their natural environment. The dust-free peels were allowed to dry under shade in the botany laboratory for 20 days. The dried peels were powdered using an electric blender. Finally, fine powder was collected from the powdered peels by sieving through a kitchen strainer and used for extraction. The Salvia officinalis leaf aqueous extract was prepared by adding $20 \mathrm{~g}$ of dried peel powder in $500 \mathrm{ml}$ of boiling distilled water for $5 \mathrm{~min}$. Then the mixture was centrifuged at $5000 \mathrm{rpm}$ for $15 \mathrm{~min}$ in a Jouan-type centrifuge, and the supernatant was filtered in flasks to obtain a cell aqueous extract and stored at $4^{\circ} \mathrm{C}$ in a refrigerator for further use [20-23].

\section{Synthesis of silver nanoparticles}

The green synthesis of the silver nanoparticles was initiated with a reaction mixture of $100 \mathrm{ml}$ of silver salt $\left(\mathrm{AgNO}_{3}\right)$ in the concentration of $1 \times$ $10^{-3} \mathrm{M}$ and $200 \mathrm{ml}$ of aqueous extract solution of Salvia officinalis leaf $(20 \mu \mathrm{g} / \mathrm{ml})$ in the proportion $1: 10$ in a conical flask. The reaction mixture was kept under magnetic stirring for $12 \mathrm{~h}$ at room temperature. At the end of the reaction time, a black colloidal solution of $\mathrm{Ag}$ was formed. The mixture was centrifuged at $10000 \mathrm{rpm}$ for $15 \mathrm{~min}$. The precipitate was washed three times with water and subsequently centrifuged $[9,10]$.

\section{Chemical characterization of silver nanoparticles}

For analysis of silver nanoparticles, common techniques of organic chemistry, i.e. SEM, FT-IR and UV-Vis. spectroscopy, were used.

The morphological features of silver nanoparticles in terms of surface area, shape and size were exactly analyzed by common morphological teste i.e., SEM (Fe-SEM ZEISS EVO18) microscopic techniques.

The biomolecules involved in the reduction of silver nanoparticles were detected using an FT-IR spectrophotometer (Shimadzu IR affinity.1). Silver nanoparticles were primarily confirmed using UVVis spectroscopy at a scan range of 200-800 nm wavelength (Jasco V670 Spectrophotometer).

\section{Determination of anti-human ovarian cancer effects of silver nanoparticles}

In this research, the human ovarian cancer cell lines i.e., Caov-3, SK-OV-3, and PA-1, and the normal cell line, i.e., HUVEC, were used to investigate the cytotoxicity and anti-human ovarian cancer effects of the $\mathrm{AgNO}_{3}$, Salvia officinalis leaf aqueous extract, and silver nanoparticles using a common cytotoxicity test, i.e., MTT assay.

For culturing the above cells, penicillin, streptomycin, and Dulbecco's modified Eagle's medium (DMEM) were used. The distribution of cells was 10,000 cells/well in 96-well plates. Then, all samples were transferred to a humidified incubator with $5 \% \mathrm{CO}_{2}$ at $37^{\circ} \mathrm{C}$. After $24 \mathrm{~h}$ incubation, all cells were treated with several concentrations i.e., 0-1000 $\mu \mathrm{g} / \mathrm{ml}$ of $\mathrm{AgNO}_{3}$, Salvia officinalis leaf aqueous extract, and silver nanoparticles, then incubated for $24 \mathrm{~h}$. $\mathrm{AgNO}_{3}$, Salvia officinalis leaf aqueous extract, and silver nanoparticles were sterilized using ultraviolet radiation for $2 \mathrm{~h}$. Finally, $5 \mathrm{mg} / \mathrm{ml}$ of MTT was added to all wells and all samples of $\mathrm{AgNO}_{3}$, Salvia officinalis leaf aqueous extract, and silver nanoparticles were transferred to an incubator at $37^{\circ} \mathrm{C}$ for $4 \mathrm{~h}$. The percentage of cell viability of samples was measured at the absorbance of 570 $\mathrm{nm}$ and according to the following formula [22]: Cell viabilty $=($ Sample $A /$ Control $A) \times 100$.

\section{Determination of the antioxidant property of silver nanoparticles}

In this method, DPPH was used as the standard as a stable radical compound. Thus, $50 \mu$ of different concentrations of $1-1000 \mu \mathrm{g} / \mathrm{ml}$ of nanoparticle was added to $5 \mathrm{ml}$ of $0.004 \%$ DPPH solution in methanol. After $30 \mathrm{~min}$ of incubation at room tem- 
perature, the samples were read against blank at $517 \mathrm{~nm}$. The percentage of inhibition of DPPH free radicals was calculated using the following formula [23]: Inhibition $(\%)=($ Sample A/Control A $) \times 100$.

In this test, the ability of hydrogen or electron atoms to be given by a nanoparticle is measured by the amount of decolorization of purple solutions 2 and 2-diphenyl-1-picryl hydrazyl or DPPH in methanol. Then the concentration of the nanoparticle with a radical inhibition percentage of 50 was calculated by the graph. Obviously, the smaller this number, the greater the antioxidant power or inhibition of free radicals. In this experiment, the synthetic antioxidant butylated hydroxytoluene was used as a positive control and all experiments were repeated three times [23].

\section{Ethics statement}

This research was approved by Shaanxi Provincial People's Hospital animal ethical committee, Approved No. SXSRM210428.

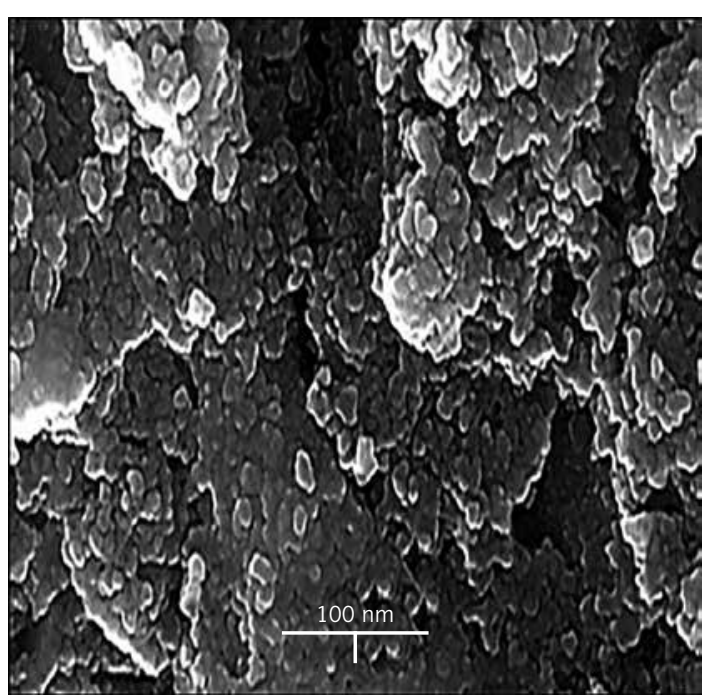

Figure 1. SEM image of AgNPs

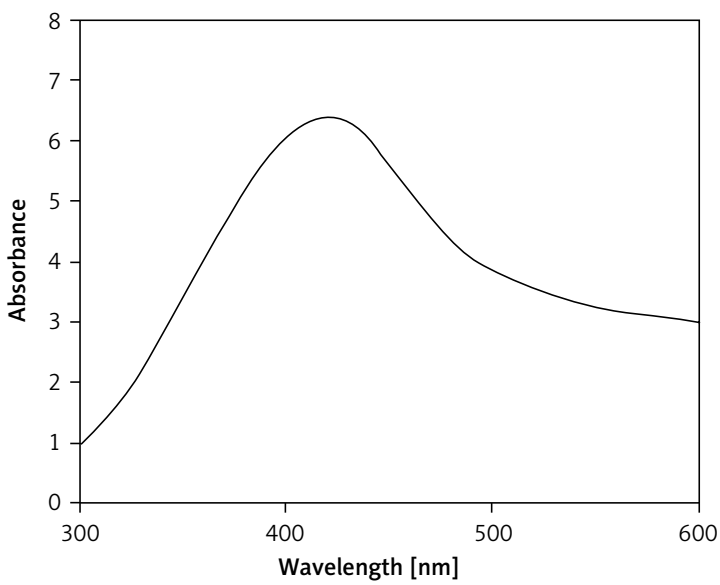

Figure 2. UV-Vis analysis of AgNPs

\section{Statistical analysis}

The obtained results were fed into SPSS-22 software and analyzed by one-way ANOVA, followed by the Duncan post-hoc test $(p \leq 0.01)$.

\section{Results and discussion}

In the present study, silver nanoparticles were prepared and synthesized in aqueous medium using Salvia officinalis leaf extract as a reducing and stabilizing agent. In addition, we investigated the cytotoxicity and anti-human ovarian cancer potentials of these nanoparticles against common human ovarian cancer cell lines, i.e. Caov-3, SK-OV-3, and PA-1 cell lines.

\section{Chemical characterization of silver nanoparticles}

The morphology of the synthesized silver nanostructures was studied by recording the SEM image of synthesized silver nanoparticles, which enumerated the formation of homogeneous and relatively spherical silver nanoparticles by stabilizing and capping agents (Figure 1). In our review of literature, different sizes in the range $10-50 \mathrm{~nm}$ have been reported for the biosynthesized silver nanoparticles using plant extracts [9, 10, 24-26].

At the beginning of the experiment, the light color of the solution immediately changed to black with a maximum wavelength of around $421 \mathrm{~nm}$, indicating the formation of silver nanoparticles as characterized by the UV-Vis spectrum (Figure 2). The silver nanoparticles produced were collected by centrifugation for further analysis. We suppose that the phenolic and triterpenoid compounds present inside the plant's biological matrix can act as stabilizing agents to prevent aggregation of $\mathrm{Ag}$ clusters through the ion-dipole intermolecular forces.

Mohammadi et al. observed the peak of silver nanoparticles containing Phoenix dactylifera seed ethanolic extract at the wavelength of

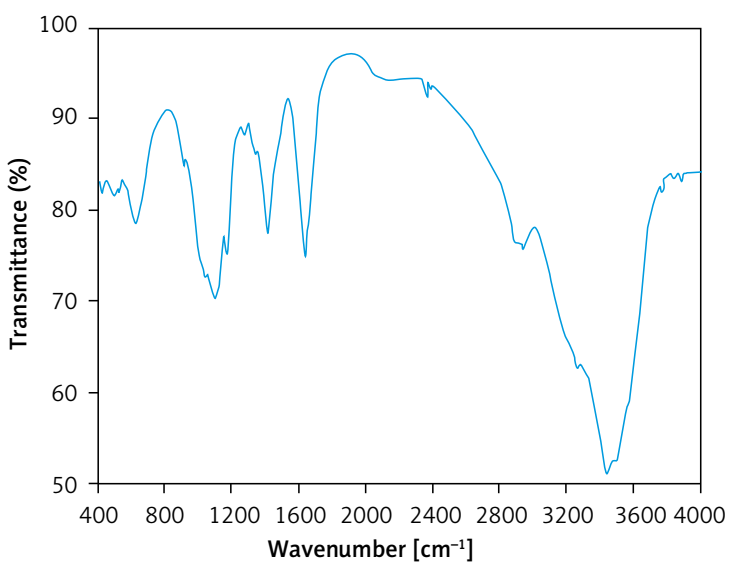

Figure 3. FT-IR analysis of AgNPs 
$438 \mathrm{~nm}[26,27]$. The Salvia officinalis leaf aqueous extract mediated synthesis of silver nanoparticles showed excellent stability even after 25 days and no considerable changes occurred in UV absorbance.

To identify the constituent responsible for the stabilization of silver nanoparticles, FT-IR was performed on biosynthesized silver nanoparticles (Figure 3). The $496 \mathrm{~cm}^{-1}$ absorption band is relevant to the Ag-O functional group resonance. This confirms that nano-sized silver particles are present in the nanocomposite. An intense and thick band emerged in the $3000-3600 \mathrm{~cm}^{-1}$ region, matching the hydroxyl functional groups stretching mode. $\mathrm{Sp}^{2}$-Carbon groups are generally the reason for the band around $1407 \mathrm{~cm}^{-1}$, while carbonyl functional groups include the $1631 \mathrm{~cm}^{-1}$ band.

\section{Cytotoxicity and anti-human ovarian cancer potential of silver nanoparticles}

The morphological parameters of silver nanoparticles which affect anticancer properties of these nanoparticles against several cancer cell lines are size, form, and surface coating. Among

A

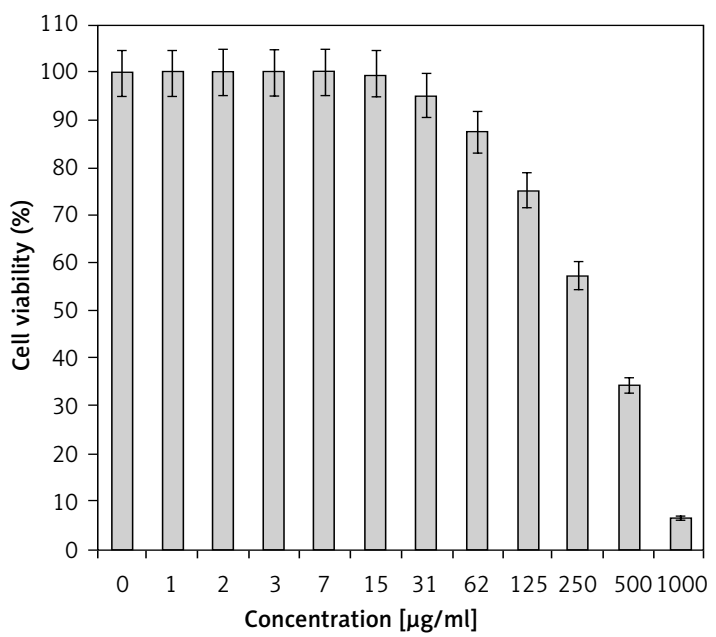

C

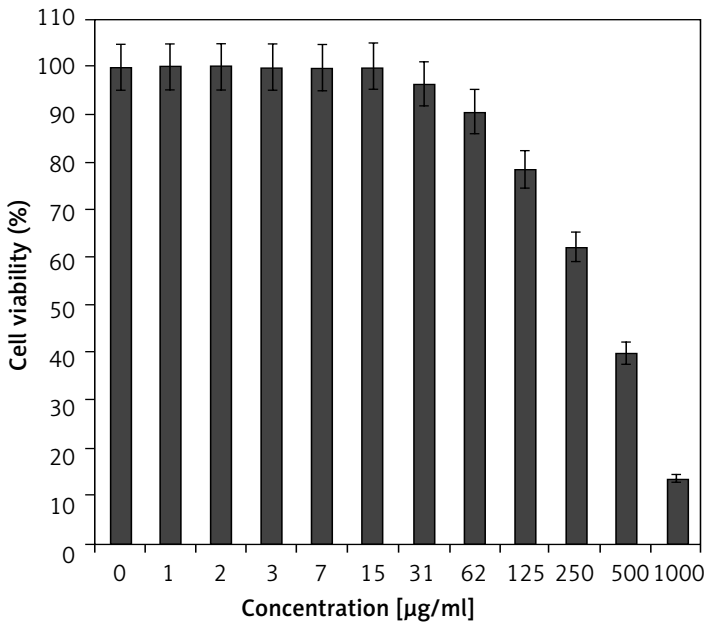

the above parameters, the role of size of silver nanoparticles is the greatest [28]. Previously, it was found that whatever the size of silver nanoparticles reduced, the ability of these nanoparticles for transferring to the cancer cell lines and killing them increased [28].

As can be observed in Figure 1, in our study the sizes of silver nanoparticles synthesized by Salvia officinalis leaf aqueous extract are in the range of 10-38 nm. Similar studies have found that silver nanoparticles at the size of $50 \mathrm{~nm}$ and lower have significant roles in removing tumor cell lines [24-27].

In the present research, the cells treated with several concentrations of $\mathrm{AgNO}_{3}$, Salvia officinalis leaf aqueous extract, and silver nanoparticles were examined by MTT test for $48 \mathrm{~h}$ regarding the cytotoxic properties on normal (HUVEC) and common human ovarian cancer (Caov-3, SK-OV-3, and PA-1) cell lines (Figure 4). The absorbance rate was determined at $570 \mathrm{~nm}$, which indicated extraordinary viability on a normal cell line (HUVEC) even up to $1000 \mu \mathrm{g} / \mathrm{ml}$ for $\mathrm{AgNO}_{3}$, Salvia officinalis leaf aqueous extract, and silver nanoparticles.

\section{B}

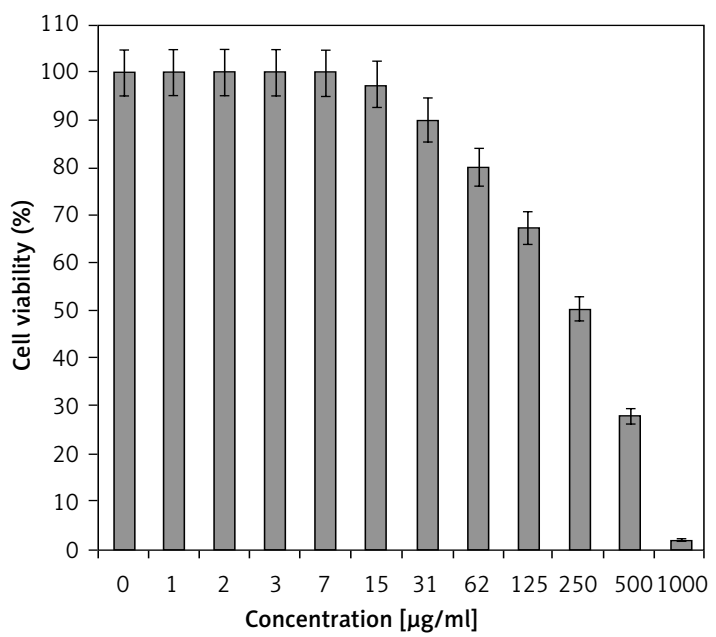

Figure 4. Anti-human ovarian cancer properties of Ag nanoparticles against Caov-3 (A), SK-OV-3 (B), and PA-1 (C) cell lines 
Table I. IC50 of Ag nanoparticles in the anti-human ovarian cancer tests

\begin{tabular}{|lc|}
\hline Cells & Ag nanoparticles $[\mu \mathrm{g} / \mathrm{ml}]$ \\
\hline $\begin{array}{l}\text { Human ovarian cancer } \\
\text { cells }\end{array}$ \\
\hline Caov-3 & 319 \\
\hline SK-OV-3 & 253 \\
\hline PA-1 & 386 \\
\hline
\end{tabular}

In the case of human ovarian cancer cell lines, their viability decreased dose-dependently in the presence of $\mathrm{AgNO}_{3}$, Salvia officinalis leaf aqueous extract, and silver nanoparticles. The $\mathrm{IC}_{50}$ of Salvia officinalis leaf aqueous extract and silver nanoparticles against ovarian cancer cell lines was less than $500 \mu \mathrm{g} / \mathrm{ml}$. The best results of cytotoxicity and anti-human ovarian cancer potential of silver nanoparticles against the above cell lines were seen in the case of the SK-OV-3 cell line (Table I).

\section{Antioxidant properties of silver nanoparticles}

In recent years, researchers evaluated plants and bio-mediated synthesized nanoparticles for antioxidant activity. Green-synthesized silver nanoparticles exhibit higher antioxidant activity for the formation of free radicals in the living system $[9,10]$. Silver nanoparticles have redox properties and play a significant role in deactivating free radicals in the living system [24-27].

In our study, the antioxidant effects of the silver nanoparticles synthesized using Salvia officinalis leaf aqueous extract were evaluated by DPPH assay, revealing concentration-dependent effects, i.e., an increase in the concentration of the silver nanoparticles leads to an increase in antioxidant activities. In the concentrations studied, the best result was seen in the high concentration or 1000 $\mu \mathrm{g} / \mathrm{ml}$ (Figure 5).

Comparative analysis of the individual antioxidant assays showed significant variations in the exertion of radical scavenging effects. Among all materials tested $\left(\mathrm{AgNO}_{3}\right.$, Salvia officinalis leaf aqueous extract, and silver nanoparticles), the silver nanoparticles indicated superior inhibition effects against DPPH. The standard (butylated hydroxytoluene) demonstrated similar antioxidant effects compared to the silver nanoparticles. The exact IC ${ }_{50}$ values of butylated hydroxytoluene and silver nanoparticles were 208 and $251 \mu \mathrm{g} / \mathrm{ml}$, respectively (Figure 5).

The anticancer effects of silver nanoparticles green-synthesized by medicinal plants have been confirmed in previous studies [9, 10, 29-31]. The study of Suman et al. clarified the anti-cervix cancer effects of silver nanoparticles containing a nat-

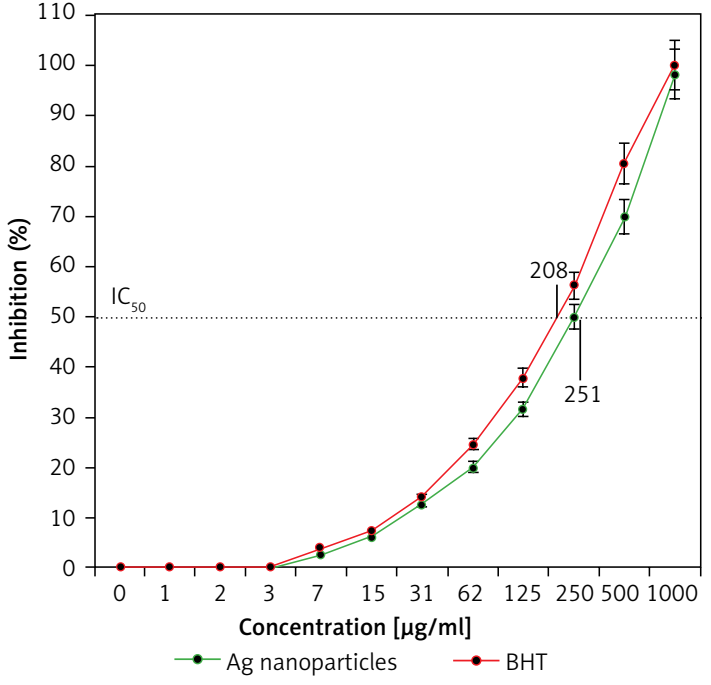

Figure 5. Antioxidant properties of $\mathrm{Ag}$ nanoparticles and BHT against DPPH

ural compound (Morinda citrifolia) against the HeLa cell line. In a previous study, silver nanoparticles killed all HeLa cells in high doses [29]. In another study, the anti-liver cancer properties of silver nanoparticles containing Piper longum leaf against Hep-2 cell lines were proved [30]. A previous study indicated that silver nanoparticles green-synthesized by Annona squamosa leaf have excellent anti-breast cancer potentials against the MCF-7 cell line [31]. Zangeneh et al. introduced a chemotherapeutic drug formulated by silver nanoparticles containing Melissa officinalis for the treatment of several types of blood cancer [9]. Also, a similar study showed that silver nanoparticles green-synthesized by Spinacia oleracea L. had an excellent anti-acute myeloid leukemia effect. In previous research, silver nanoparticles significantly removed leukemia cell lines and regulated the histopathological, immunological, biochemical, and hematological parameters in the animals [10].

The reason behind the antioxidant activity of green or biosynthesized nanoparticles could be the presence of metabolite compounds [32-35]. Also, many researchers have reported that phenolic and flavonoids attached to the nanoparticles exhibited antioxidant activity. Previously it has been reported that Salvia officinalis leaf is rich in antioxidant compounds [21]. Several studies have been carried out in the nanotechnology field using various medicinal plants, but still no report is available on silver nanoparticles synthesized using Salvia officinalis leaf aqueous extract [6-10].

Likely, the significant anti-human ovarian cancer potential of silver nanoparticles synthesized by Salvia officinalis leaf aqueous extract against common human ovarian cancer (Caov-3, SK-OV-3, and PA-1) cell lines is linked to their antioxidant activities. Similar research has revealed that anti- 
oxidant materials such as metallic nanoparticles especially silver nanoparticles and ethno-medicinal plants reduce the volume of tumors by removing free radicals [36].

The high presence of free radicals in normal cells leads to many mutation in their DNA and RNA, destroys their gene expression and then accelerates the proliferation and growth of abnormal cells or cancerous cells $[37,38]$. The high presence of free radicals in all cancers such as breast, gallbladder, stomach, rectal, liver, gastrointestinal stromal, esophageal, bile duct, small intestine, pancreatic, colon, parathyroid, thyroid, bladder, prostate, testicular, fallopian tube, vaginal, ovarian, hypopharyngeal, throat, lung, and skin cancers indicates the significant role of these molecules in angiogenesis and tumorigenesis [38, 39]. Many researchers have reported that silver nanoparticles synthesized by ethno-medicinal plants have a remarkable role in removing free radicals and growth inhibition of all cancerous cells $[39,40]$.

In conclusion, in the present study, silver nanoparticles were appropriately characterized and confirmed using UV-visible spectroscopy (UV-Vis), Fourier transformed infrared spectroscopy (FT IR), and scanning electron microscopy (SEM). The results of the organic chemistry techniques indicated that these nanoparticles had been synthesized as the best possible. Ag nanoparticles revealed significant antioxidant and cytotoxicity activities against common human ovarian cancer cell lines, i.e., Caov-3, SK-OV-3, and PA-1, without any cytotoxic effect on the normal cell line (HUVEC). After confirming these results in clinical trial studies, silver nanoparticles can be used as a chemotherapeutic drug for the treatment of ovarian cancer in humans.

\section{Acknowledgments}

This project was supported by Researchers Supporting Project number (RSP-2021/230) King Saud University, Riyadh, Saudi Arabia.

\section{Conflict of interest}

The authors declare no conflict of interest.

\section{References}

1. GBD 2015 Disease and Injury Incidence and Prevalence Collaborators. Global, regional, and national incidence, prevalence, and years lived with disability for 310 diseases and injuries, 1990-2015: a systematic analysis for the Global Burden of Disease Study 2015. Lancet 2016; 388: 1545-602.

2. GBD 2015 Mortality and Causes of Death Collaborators. Global, regional, and national life expectancy, all-cause mortality, and cause-specific mortality for 249 causes of death, 1980-2015: a systematic analysis for the Global Burden of Disease Study 2015. Lancet 2016; 388: 1459-544.
3. Ebell MH, Culp MB, Radke TJ. A systematic review of symptoms for the diagnosis of ovarian cancer. Am J Prev Med 2016; 50: 384-94.

4. Grossman DC, Curry SJ, Owens DK, et al. Screening for Ovarian Cancer: US Preventive Services Task Force Recommendation Statement. JAMA 2018; 319: 588-94.

5. Gibson SJ, Fleming GF, Temkin SM, Chase DM. The application and outcome of standard of care treatment in elderly women with ovarian cancer: a literature review over the last 10 years. Front Oncol 2016; 6: 63.

6. Zangeneh MM. Green synthesis and formulation a modern chemotherapeutic drug of Spinacia oleracea $L$. leaf aqueous extract conjugated silver nanoparticles; chemical characterization and analysis of their cytotoxicity, antioxidant, and anti-acute myeloid leukemia properties in comparison to doxorubicin in a leukemic mouse model. Appl Organometal Chem 2020; 34: e5295.

7. Ahmeda A, Zangeneh A, Zangeneh MM. Preparation, formulation, and chemical characterization of silver nanoparticles using Melissa officinalis leaf aqueous extract for the treatment of acute myeloid leukemia in vitro and in vivo conditions. Appl Organometal Chem 2020; 34: e5378.

8. Zangeneh MM. Green synthesis and chemical characterization of silver nanoparticles from aqueous extract of Falcaria vulgaris leaves and assessment of their cytotoxicity and antioxidant, antibacterial, antifungal and cutaneous wound healing properties. Appl Organometal Chem 2019; 33: e4963.

9. Zangeneh A, Zangeneh MM. Preparation, characterization, and assessment of cytotoxicity, antioxidant, antibacterial, antifungal, and cutaneous wound healing properties of titanium nanoparticles using aqueous extract of Ziziphora clinopodioides Lam leaves. Appl Organometal Chem 2019; 33: e5290.

10. Mahdavi B, Paydarfard S, Zangeneh MM, Goorani S, Seydi N, Zangeneh A. Assessment of antioxidant, cytotoxicity, antibacterial, antifungal, and cutaneous wound healing activities of green synthesized manganese nanoparticles using Ziziphora clinopodioides Lam leaves under in vitro and in vivo condition. Appl Organometal Chem 2019; 33: e5248.

11. El-Sayed IH, Huang X, El-Sayed MA. Selective laser photo-thermal therapy of epithelial carcinoma using anti-EGFR antibody conjugated gold nanoparticles. Cancer Lett 2006; 239: 129-35.

12. Chatterjee AK, Sarkar RK, Chattopadhyay AP, Aich P, Chakraborty R. A simple robust method for synthesis of metallic copper nanoparticles of high antibacterial potency against E. coli. Nanotechnology 2012; 23: 085103.

13. Azizi M, Ghourchian H, Yazdian F, Dashtestani F, AlizadehZeinabad H. Cytotoxic effect of albumin coated copper nanoparticle on human breast cancer cells of MDA-MB 231. PLoS One 2017; 12: e0188639.

14. Ramaswamy SVP, Narendhran S, Sivaraj R. Potentiating effect of ecofriendly synthesis of copper oxide nanoparticles using brown alga: antimicrobial and anticancer activities. Bull Mater Sci 2015; 39: 361-4.

15. Sumathi S, Dharani B, Sivaprabha J, Raj KS, Padma PR. Inter J Pharma Sci Inven 2013; 2: 21-6.

16. Roberson M, Rangari V, Jeelani S, Samuel T, Yates C. Synthesis and characterization silver, zinc oxide and hybrid silver/zinc oxide nanoparticles for antimicrobial applications. Nano Life 2014; 4: 1440003.

17. Gopinath M, Subbaiya R, Masilamani Selvam $M$, Suresh D. Synthesis of copper nanoparticles from ner- 
ium oleanderleaf aqueous extract and its antibacterial activity. Int J Curr Microbiol App Sci 2014; 3: 814-8.

18. Lee H, Song JY, Kim BS. Biological synthesis of copper nanoparticles using Magnolia kobus leaf extract and their antibacterial activity. J Chem Technol Biotechnol 2013; 88: 1971-7.

19. Chung IM, Rahuman AA, Marimuthu S, Kirthi AV, Anbarasan $K$. Green synthesis of copper nanoparticles using Eclipta prostrata leaves extract and their antioxidant and cytotoxic activities. Exp Ther Med 2017; 14: 18-24.

20. Jayaprakasha GK, Jagan Mohan Rao L. Chemistry, biogenesis, and biological activities of Cinnamomum zeylanicum. Crit Rev Food Sci Nutr 2011; 51: 547-62.

21. Wani MC, Taylor HL, Wall ME, Coggon P, McPhail AT. Plant antitumor agents. VI. The isolation and structure of taxol, a novel antileukemic and antitumor agent from Taxus brevifolia. J Am Chem Soc 1971; 93: 2325-7.

22. Arulmozhi V, Pandian K, Mirunalini S. Ellagic acid encapsulated chitosan nanoparticles for drug delivery system in human oral cancer cell line (KB). Colloids Surf B Biointerfaces 2013; 110: 313-20.

23. Sanchez-Moreno C, Larrauri JA, Saura-Calixto F. A procedure to measure the antiradical efficiency of polyphenols. J Sci Food Agric 1998; 76: 270-6.

24. Zangeneh MM, Bovandi S, Gharehyakheh S, Zangeneh A, Irani $P$. Green synthesis and chemical characterization of silver nanoparticles obtained using Allium saralicum aqueous extract and survey of in vitro antioxidant, cytotoxic, antibacterial and antifungal properties. App Organometal Chem 2019; 33: e4961.

25. Zangeneh A, Zangeneh MM, Moradi R. Ethnomedicinal plant-extract-assisted green synthesis of iron nanoparticles using Allium saralicum extract and their antioxidant, cytotoxicity, antibacterial, antifungal, and cutaneous wound healing activities. Appl Organometal Chem 2020; 34: e5247.

26. Mohammadi G, Zangeneh MM, Zangeneh A, Siavosh Haghighi ZM. Chemical characterization and anti-breast cancer effects of silver nanoparticles using Phoenix dactylifera seed ethanolic extract on 7,12-dimethylbenz[a] anthracene-induced mammary gland carcinogenesis in Sprague Dawley male rats. Appl Organometal Chem 2020; 34: e5136.

27. Zangeneh MM, Zangeneh A, Pirabbasi E, Moradi R, Almasi M. Falcaria vulgaris leaf aqueous extract mediated synthesis of iron nanoparticles and their therapeutic potentials under in vitro and in vivo condition. Appl Organometal Chem 2019; 33: e5246.

28. Namvar F, Rahman HS, Mohamad R, et al. Cytotoxic effect of magnetic iron oxide nanoparticles synthesized via seaweed aqueous extract. Int I Nanomedicine 2014; 19: 2479-88.

29. Suman T, Rajasree SR, Kanchana A, Elizabeth SB. Biosynthesis, characterization and cytotoxic effect of plant mediated silver nanoparticles using Morinda citrifolia root extract. Colloids Surf B Biointerfaces 2013; 106: 74-8.

30. Jacob SJP, Finub JS, Narayanan A. Synthesis of silver nanoparticles using Piper longum leaf extracts and its cytotoxic activity against Hep-2 cell line. Colloids Surf B Biointerfaces 2012; 91: 212-4.

31. Vivek R, Thangam R, Muthuchelian K, Gunasekaran P, Kaveri K, Kannan S. Green biosynthesis of silver nanoparticles from Annona squamosa leaf extract and its in vitro cytotoxic effect on MCF-7 cells. Process Biochem 2012; 47: 2405-10.
32. Oganesvan G, Galstyan A, Mnatsakanyan V, Paronikyan R, Ter-Zakharyan YZ. Phenolic and flavonoid compounds of Ziziphora clinopodioides. Chem Nat 1991; 27: 247.

33. Jeong SC, Koyyalamudi SR, Jeong YT, Song CH, Pang G. Macrophage immunomodulating and antitumor activities of polysaccharides isolated from Agaricus bisporus white button mushrooms. J Med Food 2012; 15: 58-65.

34. Rehana D, Mahendiran D, Kumar RS, Rahiman AK. Evaluation of antioxidant and anticancer activity of copper oxide nanoparticles synthesized using medicinally important plant extracts. Biomed Pharmacother 2017; 89: 1067-77.

35. Gultekin DD, Gungor AA, Onem H, Babagil A, Nadaroglu $\mathrm{H}$. Synthesis of copper nanoparticles using a different method: determination of its antioxidant and antimicrobial activity. J Turk Chem Soc A Chem 2016; 3 : 623-36.

36. Sankar R, Maheswari R, Karthik S, Shivashangari KS, Ravikumar V. Anticancer activity of Ficus religiosa engineered copper oxide nanoparticles. Mat Sci Eng C 2014; 44: 234-9.

37. Radini IA, Hasan N, Malik MA, Khan Z. Biosynthesis of iron nanoparticles using Trigonella foenum-graecum seed extract for photocatalytic methyl orange dye degradation and antibacterial applications. J Photochem Photobiol B 2018; 183: 154-63.

38. Beheshtkhoo N, Kouhbanani MAJ, Savardashtaki A, Amani AM, Taghizadeh S. Green synthesis of iron oxide nanoparticles by aqueous leaf extract of Daphne mezereum as a novel dye removing material. Appl Phys A 2018; 124: 363-9.

39. Sangami S, Manu B. Synthesis of green iron nanoparticles using laterite and their application as a Fenton-like catalyst for the degradation of herbicide Ametryn in water. Environ Technol Innov 2017; 8: 150-63.

40. Katata-Seru L, Moremedi T, Aremu OS, Bahadur I. Green synthesis of iron nanoparticles using Moringa oleifera extracts and their applications: Removal of nitrate from water and antibacterial activity against Escherichia coli. J Mol Liq 2018; 256: 296-304 